\title{
Effect of Food Attractants and Insecticide Toxicity for the Control of Spodoptera frugiperda (Lepidoptera: Noctuidae) Adults
}

\author{
Wagner Justiniano ${ }^{1} \&$ Marcos Gino Fernandes $^{1}$ \\ ${ }^{1}$ Programa de Pós-Graduação em Entomologia e Conservação da Biodiversidade, Faculdade de Ciências \\ Biológicas e Ambientais, Universidade Federal da Grande Dourados, Dourados, MS, Brazil \\ Correspondence: Wagner Justiniano, Programa de Pós-Graduação em Entomologia e Conservação da \\ Biodiversidade, Faculdade de Ciências Biológicas e Ambientais, Universidade Federal da Grande Dourados, \\ Dourados, MS, Brazil. E-mail: wagner.justiniano@bayer.com
}

Received: October 9, 2019

doi:10.5539/jas.v12n1p129

\author{
Accepted: November 8, $2019 \quad$ Online Published: December 15, 2019 \\ URL: https://doi.org/10.5539/jas.v12n1p129
}

\begin{abstract}
An alternative for the population suppression of Spodoptera frugiperda is the use of toxic baits. The objective of this study was to evaluate the dietary preference and toxic effect of insecticides associated with the attractiveness of $S$. frugiperda adults as a pest management method. The following attractions were tested: 1) $5 \%$ sucrose solution, 2) $10 \%$ sugarcane syrup, 3) $10 \%$ honey, 4) $5 \%$ hydrolyzed protein, 5) Noctovi ${ }^{\circledR} 43 \mathrm{sb}$, 6) Noctovi ${ }^{\circledR}$ OVI PLU 1-3, 7) Noctovi ${ }^{\circledR}$ OVI PHE/PAL 50-50 in the study with choice. Attractions: 1) sugarcane syrup 10\%, 2) Noctovi $^{\circledR} 43 \mathrm{sb}$, 3) Noctovi ${ }^{\circledR} 43 \mathrm{sb}+$ sugarcane syrup $10 \%$, 4) Noctovi ${ }^{\circledR}$ OVI PLU 1-3 in the study with no chance of choice. For the toxicity study, the food attraction associated with insecticides was used: methomyl $2 \%$, lambda-cyhalothrin $1 \%$, chlorpyriphos $2 \%$, spinosad $1 \%$, chlorantraniliprole $2 \%$ and chlorfenapyr $2 \%$. The experimental design was the completely randomized design (CRD) with chance of choice, without chance of choice and toxicity. The following parameters were evaluated: number of insects that fed; time in minutes that remained in the attractive food and mortality. The molasses $(10 \%)$ and Noctovi ${ }^{\circledR} 43 \mathrm{sb}$ food attractiveness were significantly more efficient in relation to feeding time and the highest number of landings was observed in the Noctovi $^{\circledR} 43 \mathrm{sb}$ treatment, both in females and total adults. Methomyl, lambda-cyhalothrin and spinosad insecticides associated with food attractiveness are promising toxic baits for the management of S. frugiperda.
\end{abstract}

Keywords: attracts and kills, toxic bait, alternative control, integrated pest management

\section{Introduction}

The monitoring is the fundamental step in the implementation of an Integrated Pest Management (IPM) program. Controlling the pest at the right time and with the right product can lead to money savings, make the target pest control program more efficient, and help preserve beneficial organisms on the farm. There are many methods of monitoring pest insects, one of which is by monitoring adult insects using appropriate traps. Such traps are considered useful tools in IPM if used correctly (Cruz et al., 2010).

The fall armyworm Spodoptera frugiperda, (J.E. Smith, 1797) (Lepidoptera: Noctuidae) is the most important pest in maize cultivation, and because it is polyphagous, it uses several commonly occurring hosts in different agroecosystems. Casmuz et al. (2010) reported the occurrence of this species in approximately 186 plant species belonging to 42 different families, in their distribution and occurrence zone.

The losses caused by the pest can cause a reduction in grain yield of up to $60 \%$, the damage resulting from the attack can occur from the emergence phase of the seedlings to the reproductive phase of the crop (Cruz, 2008; Cruz et al., 2013).

The chemical control and the use of genetically modified plants (Bt plants) are the main techniques currently used, however it has shown control failures due to the resistance of insects to insecticides and Bt proteins (Carvalho et al., 2013; Goulart et al., 2015). Thus, the use of different management strategies for fall armyworm control should be employed, aiming at reducing caterpillar infestations and consequently crop damage.

Food attractants and semi-chemicals have been investigated as alternatives for the behavioral management of different pests in plants. The chemical substances that indicate the presence of nutrient in them are in many cases, secondary compounds of plants that stimulate chemoreceptor cells, located in the taste cells of the tarsus, 
antennas and parts of the oral apparatus of insects, and that induce, among other actions, feeding and oviposition (Nation, 2002).

The use of semi-chemicals and food attractants in pest control actions is in accordance with the model recommended for future agriculture. It is a technique that has high specificity, not presenting any deleterious effect to the species that are not subject to control and no chemical residue is deposited in the environment or in the food produced. Among the identified semi-chemicals are sexual and aggregation pheromones, attractive compounds used in traps, floral compounds and glandular compounds, among others (Zarbin et al., 2009).

Toxic bait with food stimulants have been used for the identification and distribution of insect species, certification of a region or country as to the absence of a certain pest species (free area), and in pest species eradication and integrated management programs (Nascimento et al., 2000). Several attractions such as brown sugar, sucrose, corn hydrolyzed protein, fruit juices and wine vinegar are used in traps for insects monitoring and, when added to insecticides, they are recommended for the pests control, such as fruit flies (Nascimento et al., 2000; Gravena \& Benvenga, 2003; Benvenga et al., 2007). Synthetic pheromones, such as Noctovi ${ }^{\circledR}$, have recently been launched and have proven to be important allies for sampling and pest control (Silva et al., 2016).

Considering the possibility of developing methods to control adults of $S$. frugiperda, this study aimed to evaluate the effect of food attractants and their association with insecticides on adults of this pest under laboratory conditions.

\section{Materials and Methods}

\subsection{Fall Armyworm Adult Preparation}

The experiments were conducted at the Insect Sampling and Monitoring Laboratory of the Federal University of Grande Dourados in the city of Dourados, Mato Grosso do Sul, Brazil. The pupae of S. frugiperda were acquired from the Pragas.com company specialized in the creation and marketing of insect pests, based in the city of Piracicaba, state of São Paulo, Brazil.

The pupae were kept in controlled conditions of temperature $\left(26.5 \pm 2{ }^{\circ} \mathrm{C}\right)$, relative humidity $(75 \pm 1.5 \%)$ and photophase (12 hours).

Sexing occurred according to the methodology described by Zenker et al. (2007), conditioning adults in cages made of polyethylene tube with its ends closed with voil. The males and females were kept separated, being released as couples for the tests with chance of choice and without chance of choice, at six hours after the composition of the couples, to evaluate the toxicity of food attractions associated with insecticides.

To perform the bioassays, another room $(3 \times 5$ meters $)$ was also used on the premises of the Laboratory of Sampling and Monitoring of Insects, however it had large doors and windows, and simulating natural conditions of the environment without interference as to light, temperature and relative humidity to simulate the real field conditions. The insects were transferred 30 minutes before for previous adaptation and then released.

The release occurred at dusk near sunset and the evaluations occurred in the sequence of the release and at dawn, near and during sunrise. The climatic conditions during the tests with chance, without chance of choice and toxicity were: temperature $27.3 \pm 1.89{ }^{\circ} \mathrm{C} ; 28.2 \pm 1.01{ }^{\circ} \mathrm{C} ; 28.9 \pm 1{ }^{\circ} \mathrm{C}$ and relative humidity $88.8 \pm 4.3 \%, 83.7 \pm 5.2 \%$ and $79.5 \pm 9.0 \%$, respectively.

The bioassays with and without change of choice were composed of 6 repetitions for each treatment, and each repetition was released 5 couples of $S$. frugiperda with 48 to 72 hours of age not fed for a period of 24 hours, except for the toxicity test, where each couple was one repetition and 8 repetitions were used.

\subsection{Choice Test}

For the first food attractants study with a chance to choose, the experimental design was the completely randomized design (CRD), with 7 treatments and 6 repetitions. The treatments corresponded to the following attractions: 1) 5\% sucrose solution, 2) 10\% sugarcane syrup, 3) 10\% honey, 4) 5\% hydrolyzed protein, 5) Noctovi $\left.{ }^{\circledR} 43 \mathrm{sb}, 6\right)$ Noctovi $^{\circledR}$ OVI PLU 1-3, 7) Noctovi ${ }^{\circledR}$ OVI PHE/PAL 50-50. The treatments were arranged in a circular arena covered with voil, one meter high by one-meter radius (3.15 meters circumference), and the treatments were placed $45 \mathrm{~cm}$ apart. For the negative control was used a treatment with distilled water.

In the second food attractants study with no chance of choice, the experimental design used was in CRD, with 4 treatments that proved promising by previous results of the first study [Attractions: 1) sugarcane syrup 10\%, 2)

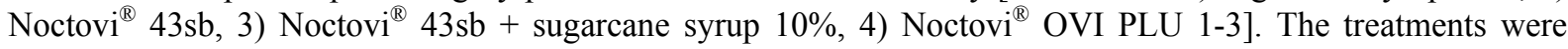
arranged in a circular arena covered with voil, one meter high by one-meter radius (3.15 meters circumference), with each feeding point (treatment) at $78 \mathrm{~cm}$ distance from each other with six repetitions performed. 
The sampling point was composed of $3 \mathrm{~cm}^{3}$ cotton pads containing $2 \mathrm{ml}$ of the solution, fixed with a wooden preacher in the upper part of the arena and visual evaluation, counting the number of male and female landings and the feeding time.

The food attractants for the tests were purchased from the manufacturers and/or authorized distributors obeying the recommendations for storage and use.

\subsection{Toxicity Test}

For the toxicity study we used the food attraction Noctovi ${ }^{\circledR} 43 \mathrm{sb}$ associated with insecticides of chemical groups and active ingredients: (Carbamate: methomyl—2\%), (Pyrethroid: lambda-cyhalothrin—1\%), (Organophosphate: chlorpyrifos-2\%), (Spinosine: spinosad-1\%), (Diamide: chlorantraniliprole-2\%) and (Pyrazole analogue: chlorfenapyr-2\%), respectively. Eight repetitions were performed, containing a couple aged 24 to 48 hours of life without food. The release occurred at dusk and the evaluations followed in the first $6 \mathrm{~h}, 12 \mathrm{~h}, 24 \mathrm{~h}, 36 \mathrm{~h}, 48 \mathrm{~h}$ and $96 \mathrm{~h}$. Each repetition was composed of a $500-\mathrm{mL}$ plastic cup (with perforated lid), the food attraction associated or not with insecticide was fixed in the upper part of the cup (lid), in a $3 \mathrm{~cm}^{3}$ cotton ball containing 2 $\mathrm{mL}$ of solution each.

The active ingredients used came from commercial insecticides available in the Brazilian market duly registered for control $S$. frugiperda.

\subsection{Statistical Analysis}

The visual evaluations were carried out according to the methodology described by France et al. (2009), separating the insects into males, females and total adults (males + females). The following parameters were evaluated: number of landings, time in minutes that remained in the attractive food and mortality due to contact with toxic baits.

The data obtained in the experiments performed, when they did not meet the normality criteria, were transformed into $\sqrt{\mathrm{x}+0.5}$ to meet the assumptions of ANOVA. Then, the averages obtained in the different treatments by the Tukey's test were compared, at a 5\% probability level. The mortality data (\%) of the treatments and the control were used to calculate the efficiency of the insecticides using the Abbott method, $\mathrm{Ma}=(\mathrm{Mt}-\mathrm{Mc}) /(100-$ $\mathrm{Mc}) \times 100$, where $\mathrm{Ma}=$ mortality corrected for the control treatment; $\mathrm{Mt}=$ mortality observed in the treatment with the insecticide and $\mathrm{Mc}=$ mortality observed in the control treatment (Abbott, 1925).

\section{Results}

Negative treatment using only distilled water was excluded from the bioassay because it did not present any attractive effect for moths.

The number of landings observed for males, females and total adults (males + females) of fall armyworm was similar for all food attractants tested: sucrose, honeydew, honey, hydrolyzed protein, Noctovi ${ }^{\circledR} 43 \mathrm{sb}$, Noctovi ${ }^{\circledR}$ OVI PLU 1-3 and Noctovi ${ }^{\circledR}$ OVI PHE/PAL. However, for the feeding time (hours) females $(32.10 \pm 7.96$; $29.68 \pm 11.16)$ and total adults $(43.49 \pm 11.26 ; 41.68 \pm 8.46)$ the treatments with honeydew and Noctovi ${ }^{\circledR} 43 \mathrm{sb}$ differed significantly from the others except Noctovi ${ }^{\mathbb{R}}$ OVI PLU 1-3, respectively (Table 1).

The attractive honeydew-based Noctovi ${ }^{\circledR} 43 \mathrm{sb}$, Noctovi ${ }^{\circledR} 43 \mathrm{sb}+$ honeydew and Noctovi ${ }^{\circledR}$ OVI PLU 1-3, subjected to the no-choice test, resembled each other in terms of the number of landings for males and feeding time for both males, females and adults. There was a higher number of landings for females and adults exposed to the food attraction Noctovi ${ }^{\circledR} 43 \mathrm{sb}$ (Table 2). 
Table 1. Effect of different food attractants with choice in the behavior of landings (mean \pm standard error) and feeding time (mean \pm standard error) of males, females and adults (males + females) of Spodoptera frugiperda under controlled laboratory conditions. UFGD, Dourados, Mato Grosso do Sul, Brazil, 2019

\begin{tabular}{|c|c|c|}
\hline Treatment & Landing $^{(1)}$ & Time (minutes) $)^{(1)}$ \\
\hline \multicolumn{3}{|l|}{ Males } \\
\hline Sucrose $(5 \%)$ & $0.50 \pm 0.22 \mathrm{~A}$ & $6.95 \pm 3.29 \mathrm{~A}$ \\
\hline Honeydew (10\%) & $1.00 \pm 0.37 \mathrm{~A}$ & $12.06 \pm 5.46 \mathrm{~A}$ \\
\hline Honey $(10 \%)$ & $1.00 \pm 0.26 \mathrm{~A}$ & $7.27 \pm 4.53 \mathrm{~A}$ \\
\hline Hydrolyzed protein $(5 \%)$ & $0.50 \pm 0.22 \mathrm{~A}$ & $0.05 \pm 0.04 \mathrm{~A}$ \\
\hline Noctovi $^{\circledR} 43 \mathrm{sb}$ & $0.67 \pm 0.33 \mathrm{~A}$ & $12.00 \pm 4.55 \mathrm{~A}$ \\
\hline Noctovi $^{(\mathbb{B}}$ OVI PLU 1-3 & $0.67 \pm 0.33 \mathrm{~A}$ & $4.22 \pm 3.07 \mathrm{~A}$ \\
\hline Noctovi ${ }^{\circledR}$ OVI PHE/PAL 50-50 & $0.33 \pm 0.21 \mathrm{~A}$ & $7.44 \pm 4.72 \mathrm{~A}$ \\
\hline C.V. $\%{ }^{(2)}$ & 31.51 & 83.26 \\
\hline \multicolumn{3}{|l|}{ Females } \\
\hline Sucrose $(5 \%)$ & $0.33 \pm 021 \mathrm{~A}$ & $3.16 \pm 1.84 \mathrm{~A}$ \\
\hline Honeydew $(10 \%)$ & $1.33 \pm 0.21 \mathrm{~A}$ & $32.10 \pm 7.96 \mathrm{~B}$ \\
\hline Honey $(10 \%)$ & $0.33 \pm 0.21 \mathrm{~A}$ & $3.93 \pm 2.69 \mathrm{~A}$ \\
\hline Hydrolyzed protein $(5 \%)$ & $0.83 \pm 0.31 \mathrm{~A}$ & $0.83 \pm 0.55 \mathrm{~A}$ \\
\hline Noctovi $^{\circledR} 43 \mathrm{sb}$ & $1.33 \pm 0.21 \mathrm{~A}$ & $29.68 \pm 11.16 \mathrm{~B}$ \\
\hline Noctovi $^{\circledR}$ OVI PLU $1-3$ & $1.00 \pm 0.33 \mathrm{~A}$ & $21.35 \pm 5.76 \mathrm{AB}$ \\
\hline Noctovi ${ }^{\circledR}$ OVI PHE/PAL 50-50 & $0.67 \pm 0.21 \mathrm{~A}$ & $2.29 \pm 1.34 \mathrm{~A}$ \\
\hline C.V. $\%{ }^{(2)}$ & 25.30 & 56.31 \\
\hline \multicolumn{3}{|l|}{ Adults (males + females) } \\
\hline Sucrose $(5 \%)$ & $0.83 \pm 0.39 \mathrm{~A}$ & $10.12 \pm 3.43 \mathrm{~A}$ \\
\hline Honeydew $(10 \%)$ & $2.33 \pm 0.51 \mathrm{~A}$ & $43.49 \pm 11.26 \mathrm{~B}$ \\
\hline Honey $(10 \%)$ & $1.33 \pm 0.21 \mathrm{~A}$ & $11.20 \pm 4.04 \mathrm{~A}$ \\
\hline Hydrolyzed protein $(5 \%)$ & $1.33 \pm 0.42 \mathrm{~A}$ & $0.94 \pm 0.53 \mathrm{~A}$ \\
\hline Noctovi $^{\circledR} 43 \mathrm{sb}$ & $2.00 \pm 0.26 \mathrm{~A}$ & $41.68 \pm 8.46 \mathrm{~B}$ \\
\hline Noctovi $^{\circledR}$ OVI PLU 1-3 & $1.67 \pm 0.61 \mathrm{~A}$ & $25.56 \pm 6.04 \mathrm{AB}$ \\
\hline Noctovi ${ }^{\circledR}$ OVI PHE/PAL 50-50 & $1.00 \pm 0.26 \mathrm{~A}$ & $9.74 \pm 4.15 \mathrm{~A}$ \\
\hline C.V. $\%(2)$ & 24.41 & 42.27 \\
\hline
\end{tabular}

Note. ${ }^{\left({ }^{(1)}\right.}$ Original data with averages followed by the same letter in the columns do not differ from each other by Tukey's test, at $5 \%$ probability. ${ }^{(2)}$ C.V. $\%$ of data transformed into $\sqrt{\mathrm{x}+0.5}$.

The attractive honeydew-based Noctovi ${ }^{\circledR} 43 \mathrm{sb}$, Noctovi ${ }^{\circledR} 43 \mathrm{sb}+$ honeydew and Noctovi ${ }^{\circledR}$ OVI PLU 1-3, subjected to the no-choice test, resembled each other in terms of the number of landings for males and feeding time for both males, females and adults. There was a higher number of landings for females and adults exposed to the food attraction Noctovi ${ }^{\circledR} 43 \mathrm{sb}$ (Table 2).

No change in adult response to attractiveness was reported, but the presence of insecticides significantly affected the survival of females, males and adults of $S$. frugiperda over time (Figure 1). The percentage of mortality varied over time among the insecticides tested, differentiating them in terms of adult control potential (Table 3 and Figure 1).

Mortality of fall armyworm, caused by insecticides, increased directly within the evaluation periods for males, females and adults (Table 3). Methomyl caused mortality of 50\% and 41.7 after one hour of observation for males and total adults. After two hours, the best results were obtained with methomyl and lambda-cylotrine, $83.3 \%, 83.3 \%, 75 \%$ and $58.2 \%$, respectively. 
Table 2. Effect of different food attractants without chance of choice in the behavior of landings (mean \pm standard error) and feeding time (mean \pm standard error) of males, females and adults (males + females) of S. frugiperda under controlled laboratory conditions. UFGD, Dourados, Mato Grosso do Sul, Brazil, 2019

\begin{tabular}{|c|c|c|}
\hline Treatment & Landing $^{(1)}$ & Time (minutes) ${ }^{(1)}$ \\
\hline \multicolumn{3}{|l|}{ Males } \\
\hline Honeydew $(10 \%)$ & $0.67 \pm 0.33 \mathrm{~A}$ & $27.57 \pm 10.87 \mathrm{~A}$ \\
\hline Noctovi $^{\circledR} 43 \mathrm{sb}$ & $1.33 \pm 0.41 \mathrm{~A}$ & $32.27 \pm 9.70 \mathrm{~A}$ \\
\hline Noctovi $^{\circledR} 43 \mathrm{sb}+$ Honeydew $(10 \%)$ & $0.50 \pm 0.27 \mathrm{~A}$ & $20.55 \pm 24.10 \mathrm{~A}$ \\
\hline Noctovi $^{\circledR}$ OVI PLU 1-3 & $1.33 \pm 0.41 \mathrm{~A}$ & $42.66 \pm 12.48 \mathrm{~A}$ \\
\hline C.V. $\%$ & 28.19 & 54.82 \\
\hline \multicolumn{3}{|l|}{ Females } \\
\hline Honeydew $(10 \%)$ & $0.50 \pm 0.34 \mathrm{~A}$ & $6.83 \pm 4.67 \mathrm{~A}$ \\
\hline Noctovi $^{\circledR} 43 \mathrm{sb}$ & $1.67 \pm 0.49 \mathrm{~B}$ & $40.07 \pm 10.10 \mathrm{~A}$ \\
\hline Noctovi ${ }^{\circledR} 43 \mathrm{sb}+$ Honeydew $(10 \%)$ & $1.17 \pm 0.32 \mathrm{AB}$ & $35.34 \pm 12.84 \mathrm{~A}$ \\
\hline Noctovi $^{\circledR}$ OVI PLU 1-3 & $1.00 \pm 0.26 \mathrm{AB}$ & $33.80 \pm 11.95 \mathrm{~A}$ \\
\hline C.V. $\%$ & 19.91 & 53.43 \\
\hline \multicolumn{3}{|l|}{ Adults (males + females) } \\
\hline Honeydew $(10 \%)$ & $1.17 \pm 0.60 \mathrm{~A}$ & $34.40 \pm 13.18 \mathrm{~A}$ \\
\hline Noctovi $^{\circledR} 43 \mathrm{sb}$ & $3.00 \pm 0.73 \mathrm{~B}$ & $72.33 \pm 13.57 \mathrm{~A}$ \\
\hline Noctovi $^{\circledR} 43 \mathrm{sb}+$ Honeydew $(10 \%)$ & $1.67 \pm 0.42 \mathrm{AB}$ & $55.89 \pm 22.02 \mathrm{~A}$ \\
\hline Noctovi $^{\circledR}$ OVI PLU $1-3$ & $2.33 \pm 0.49 \mathrm{AB}$ & $76.47 \pm 17.21 \mathrm{~A}$ \\
\hline C.V. $\%$ & 22.69 & 36.93 \\
\hline
\end{tabular}

Note. ${ }^{(1)}$ Original data with averages followed by the same letter in the columns do not differ from each other by Tukey's test, at $5 \%$ probability. ${ }^{(2)}$ C.V. \% of data transformed into $\sqrt{\mathrm{x}+0.5}$.

In the evaluation after four hours the toxic lures based on methomyl, lambda-cyhalothrin and spinosad insecticides differed significantly from the others, proving the deleterious effect for males and total adults in the order of $83.3 \%, 100 \%, 66.7 \%, 91.7 \%, 100 \%, 83.3 \%$, respectively. For females mortality was $100 \%$.

The 12 hours of observation, methomyl, lambda-cyhalothrin da-cyolotrin, chlorpyrifos, spinosad, chlorantraliniprole caused a significant reduction in the number of living males and females, after 24 hours, all the active agents differed from the control treatments and in the 48 hours they caused $100 \%$ mortality for males, females and total adults. The evaluation, 96 hours after showed that there was no mortality in the treatment containing only the food attraction in the absence of insecticides.

The active ingredients methomyl, lambda-cyhalothrin and spinosad associated with the commercial food attraction Noctovi $^{\circledR} 43 \mathrm{sb}$, promoted $100 \%$ mortality of the insect population faster than the other molecules tested. The mean time in hours observed fo mortality of S. frugiperda males, females and adults (couples) were $2.71 \pm 1.88,1.41 \pm 0.40,2.06 \pm 0.94,1.69 \pm 0.28,2.04 \pm 0.28,1.86 \pm 0.19,5.29 \pm 2.13,2.32 \pm 0.36$ and $3.80 \pm 1.12$, respectively, for three most promising actives under the conditions studied (Figure 1). 
Table 3. Mortality (mean \pm standard error) of males, females and total adults (males + females) of S. frugiperda, because of 6 insecticides/chemical groups associated with food attractants under laboratory conditions. UFGD, Dourados, Mato Grosso do Sul, Brazil, 2019

\begin{tabular}{|c|c|c|c|c|c|c|c|c|c|c|c|}
\hline \multirow{2}{*}{ Treatments } & \multicolumn{11}{|c|}{ Time (hours) ${ }^{(1)}$} \\
\hline & 0.25 & 0.5 & 1 & 2 & 4 & 6 & 12 & 24 & 36 & 48 & 96 \\
\hline \multicolumn{12}{|l|}{ Males } \\
\hline Water+honey $(10 \%)$ & $0.00 \pm 0.00 \mathrm{~A}$ & $0.00 \pm 0.00 \mathrm{~A}$ & $0.00 \pm 0.00 \mathrm{~A}$ & $0.00 \pm 0.00 \mathrm{~A}$ & $0.00 \pm 0.00 \mathrm{~A}$ & $0.00 \pm 0.00 \mathrm{~A}$ & $0.00 \pm 0.00 \mathrm{~A}$ & $0.00 \pm 0.00 \mathrm{~A}$ & $0.00 \pm 0.00 \mathrm{~A}$ & $0.00 \pm 0.00 \mathrm{~A}$ & $0.00 \pm 0.00 \mathrm{~A}$ \\
\hline Noctovi $($ $43 \mathrm{sb}$ & $0.00 \pm 0.00 \mathrm{~A}$ & $0.00 \pm 0.00 \mathrm{~A}$ & $0.00 \pm 0.00 \mathrm{~A}$ & $0.00 \pm 0.00 \mathrm{~A}$ & $0.00 \pm 0.00 \mathrm{~A}$ & $0.00 \pm 0.00 \mathrm{~A}$ & $0.00 \pm 0.00 \mathrm{~A}$ & $0.00 \pm 0.00 \mathrm{~A}$ & $0.00 \pm 0.00 \mathrm{~A}$ & $0.00 \pm 0.00 \mathrm{~A}$ & $0.00 \pm 0.00 \mathrm{~A}$ \\
\hline Methomyl (2\%) & $33.3 \pm 0.21 \mathrm{~A}$ & $33.3 \pm 0.21 \mathrm{~A}$ & $50.0 \pm 0.22 \mathrm{~B}$ & $83.3 \pm 0.17 \mathrm{~B}$ & $83.3 \pm 0.17 \mathrm{C}$ & $83.3 \pm 0.17 \mathrm{BC}$ & $100.0 \pm 0.00 \mathrm{~B}$ & $100.0 \pm 0.00 \mathrm{~B}$ & $100.0 \pm 0.00 \mathrm{~B}$ & $100.0 \pm 0.00 \mathrm{~B}$ & $100.0 \pm 0.00 \mathrm{~B}$ \\
\hline Lambda-cyhalothrin (1\%) & $0.00 \pm 0.00 \mathrm{~A}$ & $16.7 \pm 0.17 \mathrm{~A}$ & $16.7 \pm 0.17 \mathrm{AB}$ & $83.3 \pm 0.17 \mathrm{~B}$ & $100.0 \pm 0.00 \mathrm{C}$ & $100.0 \pm 0.00 \mathrm{C}$ & $100.0 \pm 0.00 \mathrm{~B}$ & $100.0 \pm 0.00 \mathrm{~B}$ & $100.0 \pm 0.00 \mathrm{~B}$ & $100.0 \pm 0.00 \mathrm{~B}$ & $100.0 \pm 0.00 \mathrm{~B}$ \\
\hline Chlorpyrifos ( $2 \%)$ & $0.00 \pm 0.00 \mathrm{~A}$ & $0.00 \pm 0.00 \mathrm{~A}$ & $0.00 \pm 0.00 \mathrm{~A}$ & $0.00 \pm 0.00 \mathrm{~A}$ & $50.0 \pm 0.22 \mathrm{ABC}$ & $50.0 \pm 0.22 \mathrm{ABC}$ & $50.0 \pm 0.22 \mathrm{AB}$ & $66.7 \pm 0.21 \mathrm{~B}$ & $100.0 \pm 0.00 \mathrm{~B}$ & $100.0 \pm 0.00 \mathrm{~B}$ & $100.0 \pm 0.00 \mathrm{~B}$ \\
\hline Spinosad (1\%) & $0.00 \pm 0.00 \mathrm{~A}$ & $0.00 \pm 0.00 \mathrm{~A}$ & $0.00 \pm 0.00 \mathrm{~A}$ & $33.3 \pm 0.21 \mathrm{AB}$ & $66.7 \pm 0.21 \mathrm{BC}$ & $66.7 \pm 0.21 \mathrm{ABC}$ & $100.0 \pm 0.00 \mathrm{~B}$ & $100.0 \pm 0.00 \mathrm{~B}$ & $100.0 \pm 0.00 \mathrm{~B}$ & $100.0 \pm 0.00 \mathrm{~B}$ & $100.0 \pm 0.00 \mathrm{~B}$ \\
\hline Chlorantraliniprole (2\%) & $0.00 \pm 0.00 \mathrm{~A}$ & $0.00 \pm 0.00 \mathrm{~A}$ & $0.00 \pm 0.00 \mathrm{~A}$ & $0.00 \pm 0.00 \mathrm{~A}$ & $0.00 \pm 0.00 \mathrm{~A}$ & $33.3 \pm 0.21 \mathrm{ABC}$ & $83.3 \pm 0.17 \mathrm{~B}$ & $100.0 \pm 0.00 \mathrm{~B}$ & $100.0 \pm 0.00 \mathrm{~B}$ & $100.0 \pm 0.00 \mathrm{~B}$ & $100.0 \pm 0.00 \mathrm{~B}$ \\
\hline Chlorfenapyr (2\%) & $0.00 \pm 0.00 \mathrm{~A}$ & $0.00 \pm 0.00 \mathrm{~A}$ & $0.00 \pm 0.00 \mathrm{~A}$ & $16.7 \pm 0.17 \mathrm{~A}$ & $16.7 \pm 0.17 \mathrm{AB}$ & $16.7 \pm 0.17 \mathrm{AB}$ & $66.7 \pm 0.21 \mathrm{~B}$ & $83.3 \pm 0.17 \mathrm{~B}$ & $83.3 \pm 0.17 \mathrm{~B}$ & $100.0 \pm 0.00 \mathrm{~B}$ & $100.0 \pm 0.00 \mathrm{~B}$ \\
\hline C.V. $\%^{(2)}$ & 12.97 & 15.56 & 16.15 & 18.56 & 19.53 & 21.26 & 15.69 & 11.58 & 6.89 & 0.00 & 0.00 \\
\hline \multicolumn{12}{|l|}{ Females } \\
\hline Water+honey $(10 \%)$ & $0.00 \pm 0.00 \mathrm{~A}$ & $0.00 \pm 0.00 \mathrm{~A}$ & $0.00 \pm 0.00 \mathrm{~A}$ & $0.00 \pm 0.00 \mathrm{~A}$ & $0.00 \pm 0.00 \mathrm{~A}$ & $0.00 \pm 0.00 \mathrm{~A}$ & $0.00 \pm 0.00 \mathrm{~A}$ & $0.00 \pm 0.00 \mathrm{~A}$ & $0.00 \pm 0.00 \mathrm{~A}$ & $0.00 \pm 0.00 \mathrm{~A}$ & $0.00 \pm 0.00 \mathrm{~A}$ \\
\hline Noctovi $(43 \mathrm{sb}$ & $0.00 \pm 0.00 \mathrm{~A}$ & $0.00 \pm 0.00 \mathrm{~A}$ & $0.00 \pm 0.00 \mathrm{~A}$ & $0.00 \pm 0.00 \mathrm{~A}$ & $0.00 \pm 0.00 \mathrm{~A}$ & $0.00 \pm 0.00 \mathrm{~A}$ & $0.00 \pm 0.00 \mathrm{~A}$ & $0.00 \pm 0.00 \mathrm{~A}$ & $0.00 \pm 0.00 \mathrm{~A}$ & $0.00 \pm 0.00 \mathrm{~A}$ & $0.00 \pm 0.00 \mathrm{~A}$ \\
\hline Methomyl (2\%) & $16.7 \pm 0.17 \mathrm{~A}$ & $16.7 \pm 0.17 \mathrm{~A}$ & $33.3 \pm 0.21 \mathrm{~A}$ & $66.7 \pm 0.21 \mathrm{~B}$ & $100.0 \pm 0.00 \mathrm{~B}$ & $100.0 \pm 0.00 \mathrm{~B}$ & $100.0 \pm 0.00 \mathrm{~B}$ & $100.0 \pm 0.00 \mathrm{~B}$ & $100.0 \pm 0.00 \mathrm{~B}$ & $100.0 \pm 0.00 \mathrm{~B}$ & $100.0 \pm 0.00 \mathrm{~B}$ \\
\hline Lambda-cyhalothrin (1\%) & $0.00 \pm 0.00 \mathrm{~A}$ & $0.00 \pm 0.00 \mathrm{~A}$ & $0.00 \pm 0.00 \mathrm{~A}$ & $33.3 \pm 0.21 \mathrm{AB}$ & $100.0 \pm 0.00 \mathrm{~B}$ & $100.0 \pm 0.00 \mathrm{~B}$ & $100.0 \pm 0.00 \mathrm{~B}$ & $100.0 \pm 0.00 \mathrm{~B}$ & $100.0 \pm 0.00 \mathrm{~B}$ & $100.0 \pm 0.00 \mathrm{~B}$ & $100.0 \pm 0.00 \mathrm{~B}$ \\
\hline Chlorpyrifos ( $2 \%)$ & $0.00 \pm 0.00 \mathrm{~A}$ & $0.00 \pm 0.00 \mathrm{~A}$ & $0.00 \pm 0.00 \mathrm{~A}$ & $0.00 \pm 0.00 \mathrm{~A}$ & $16.7 \pm 0.17 \mathrm{~A}$ & $16.7 \pm 0.17 \mathrm{~A}$ & $50.0 \pm 0.22 \mathrm{AB}$ & $83.3 \pm 0.17 \mathrm{~B}$ & $100.0 \pm 0.00 \mathrm{~B}$ & $100.0 \pm 0.00 \mathrm{~B}$ & $100.0 \pm 0.00 \mathrm{~B}$ \\
\hline Spinosad (1\%) & $0.00 \pm 0.00 \mathrm{~A}$ & $0.00 \pm 0.00 \mathrm{~A}$ & $0.00 \pm 0.00 \mathrm{~A}$ & $33.3 \pm 0.21 \mathrm{AB}$ & $100.0 \pm 0.00 \mathrm{~B}$ & $100.0 \pm 0.00 \mathrm{~B}$ & $100.0 \pm 0.00 \mathrm{~B}$ & $100.0 \pm 0.00 \mathrm{~B}$ & $100.0 \pm 0.00 \mathrm{~B}$ & $100.0 \pm 0.00 \mathrm{~B}$ & $100.0 \pm 0.00 \mathrm{~B}$ \\
\hline Chlorantraliniprole (2\%) & $0.00 \pm 0.00 \mathrm{~A}$ & $0.00 \pm 0.00 \mathrm{~A}$ & $0.00 \pm 0.00 \mathrm{~A}$ & $0.00 \pm 0.00 \mathrm{~A}$ & $16.7 \pm 0.17 \mathrm{~A}$ & $66.7 \pm 0.21 \mathrm{~B}$ & $83.3 \pm 0.17 \mathrm{~B}$ & $100.0 \pm 0.00 \mathrm{~B}$ & $100.0 \pm 0.00 \mathrm{~B}$ & $100.0 \pm 0.00 \mathrm{~B}$ & $100.0 \pm 0.00 \mathrm{~B}$ \\
\hline Chlorfenapyr (2\%) & $0.00 \pm 0.00 \mathrm{~A}$ & $0.00 \pm 0.00 \mathrm{~A}$ & $0.00 \pm 0.00 \mathrm{~A}$ & $0.00 \pm 0.00 \mathrm{~A}$ & $0.00 \pm 0.00 \mathrm{~A}$ & $0.00 \pm 0.00 \mathrm{~A}$ & $50.0 \pm 0.22 \mathrm{AB}$ & $83.3 \pm 0.17 \mathrm{~B}$ & $100 \pm 0.00 \mathrm{~B}$ & $100.0 \pm 0.00 \mathrm{~B}$ & $100.0 \pm 0.00 \mathrm{~B}$ \\
\hline C.V. $\%^{(2)}$ & 10.41 & 56.31 & 12.97 & 20.88 & 11.61 & 13.16 & 16.00 & 9.98 & 0.00 & 0.00 & 0.00 \\
\hline \multicolumn{12}{|l|}{ Adults (males+females) } \\
\hline Water+honey $(10 \%)$ & $0.00 \pm 0.00 \mathrm{~A}$ & $0.00 \pm 0.00 \mathrm{~A}$ & $0.00 \pm 0.00 \mathrm{~A}$ & $0.00 \pm 0.00 \mathrm{~A}$ & $0.00 \pm 0.00 \mathrm{~A}$ & $0.00 \pm 0.00 \mathrm{~A}$ & $0.00 \pm 0.00 \mathrm{~A}$ & $0.00 \pm 0.00 \mathrm{~A}$ & $0.00 \pm 0.00 \mathrm{~A}$ & $0.00 \pm 0.00 \mathrm{~A}$ & $0.00 \pm 0.00 \mathrm{~A}$ \\
\hline Noctovi ${ }^{\circledR} 43 \mathrm{sb}$ & $0.00 \pm 0.00 \mathrm{~A}$ & $0.00 \pm 0.00 \mathrm{~A}$ & $0.00 \pm 0.00 \mathrm{~A}$ & $0.00 \pm 0.00 \mathrm{~A}$ & $0.00 \pm 0.00 \mathrm{~A}$ & $0.00 \pm 0.00 \mathrm{~A}$ & $0.00 \pm 0.00 \mathrm{~A}$ & $0.00 \pm 0.00 \mathrm{~A}$ & $0.00 \pm 0.00 \mathrm{~A}$ & $0.00 \pm 0.00 \mathrm{~A}$ & $0.00 \pm 0.00 \mathrm{~A}$ \\
\hline Methomyl (2\%) & $25.0 \pm 0.13 \mathrm{~B}$ & $25.0 \pm 0.13 \mathrm{~B}$ & $41.7 \pm 0.15 \mathrm{~B}$ & $75.0 \pm 0.13 \mathrm{C}$ & $91.7 \pm 0.08 \mathrm{~B}$ & $91.7 \pm 0.08 \mathrm{CD}$ & $100.0 \pm 0.00 \mathrm{C}$ & $100.0 \pm 0.00 \mathrm{~B}$ & $100.0 \pm 0.00 \mathrm{~B}$ & $100.0 \pm 0.00 \mathrm{~B}$ & $100.0 \pm 0.00 \mathrm{~B}$ \\
\hline Lambda-cyhalothrin (1\%) & $0.00 \pm 0.00 \mathrm{~A}$ & $0.08 \pm 0.08 \mathrm{AB}$ & $0.08 \pm 0.08 \mathrm{~A}$ & $58.2 \pm 0.15 \mathrm{BC}$ & $100.0 \pm 0.00 \mathrm{~B}$ & $100.0 \pm 0.00 \mathrm{D}$ & $100.0 \pm 0.00 \mathrm{C}$ & $100.0 \pm 0.00 \mathrm{~B}$ & $100.0 \pm 0.00 \mathrm{~B}$ & $100.0 \pm 0.00 \mathrm{~B}$ & $100.0 \pm 0.00 \mathrm{~B}$ \\
\hline Chlorpyrifos (2\%) & $0.00 \pm 0.00 \mathrm{~A}$ & $0.00 \pm 0.00 \mathrm{~A}$ & $0.00 \pm 0.00 \mathrm{~A}$ & $0.00 \pm 0.00 \mathrm{~A}$ & $33.3 \pm 0.14 \mathrm{~A}$ & $33.3 \pm 0.14 \mathrm{AB}$ & $50.0 \pm 0.15 \mathrm{~B}$ & $75.0 \pm 0.13 \mathrm{~B}$ & $75.0 \pm 0.13 \mathrm{~B}$ & $100.0 \pm 0.00 \mathrm{~B}$ & $100.0 \pm 0.00 \mathrm{~B}$ \\
\hline Spinosad (1\%) & $0.00 \pm 0.00 \mathrm{~A}$ & $0.00 \pm 0.00 \mathrm{~A}$ & $0.00 \pm 0.00 \mathrm{~A}$ & $33.3 \pm 0.14 \mathrm{AB}$ & $83.3 \pm 0.11 \mathrm{~B}$ & $83.3 \pm 0.11 \mathrm{CD}$ & $100.0 \pm 0.00 \mathrm{C}$ & $100.0 \pm 0.00 \mathrm{~B}$ & $100.0 \pm 0.00 \mathrm{~B}$ & $100.0 \pm 0.00 \mathrm{~B}$ & $100.0 \pm 0.00 \mathrm{~B}$ \\
\hline Chlorantraliniprole (2\%) & $0.00 \pm 0.00 \mathrm{~A}$ & $0.00 \pm 0.00 \mathrm{~A}$ & $0.00 \pm 0.00 \mathrm{~A}$ & $0.00 \pm 0.00 \mathrm{~A}$ & $0.00 \pm 0.00 \mathrm{~A}$ & $50.0 \pm 0.15 \mathrm{BC}$ & $83.3 \pm 0.11 \mathrm{AB}$ & $100.0 \pm 0.00 \mathrm{~B}$ & $100.0 \pm 0.00 \mathrm{~B}$ & $100.0 \pm 0.00 \mathrm{~B}$ & $100.0 \pm 0.00 \mathrm{~B}$ \\
\hline Chlorfenapyr (2\%) & $0.00 \pm 0.00 \mathrm{~A}$ & $0.00 \pm 0.00 \mathrm{~A}$ & $0.00 \pm 0.00 \mathrm{~A}$ & $0.08 \pm 0.08 \mathrm{~A}$ & $0.08 \pm 0.08 \mathrm{~A}$ & $0.08 \pm 0.08 \mathrm{AB}$ & $58.3 \pm 0.15 \mathrm{~B}$ & $83.3 \pm 0.11 \mathrm{~B}$ & $91.7 \pm 0.08 \mathrm{~B}$ & $100.0 \pm 0.00 \mathrm{~B}$ & $100.0 \pm 0.00 \mathrm{~B}$ \\
\hline C.V. $\%{ }^{(2)}$ & 11.44 & 13.01 & 14.29 & 19.65 & 16.46 & 18.03 & 15.19 & 10.41 & 13.76 & 0.00 & 0.00 \\
\hline
\end{tabular}

Note. ${ }^{(1)}$ Original data with averages followed by the same letter in the columns do not differ from each other by Tukey's test, at $5 \%$ probability. ${ }^{(2)}$ C.V. \% of data transformed into $\sqrt{\mathrm{x}+0.5}$.

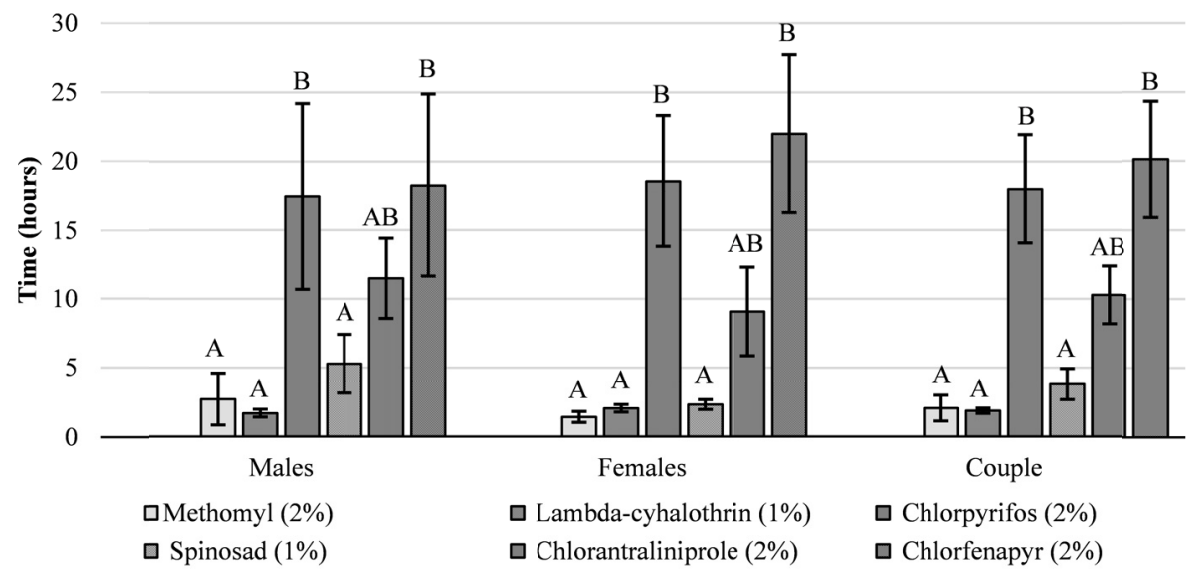

Figure 1. Time in hours for mortality (mean \pm standard error) of male, female and adult (couple) Spodoptera frugiperda under controlled laboratory conditions. UFGD, Dourados, Mato Grosso do Sul. Brazil, 2019 


\section{Discussion}

For the choice of an appropriate attraction, some factors should be considered, such as: cost, ease of application, attractiveness of adults, especially females and landing time associated with feeding, because the greater the contact of the sensory organs of insects with the bait, the greater the effectiveness of the associated insecticide (França et al., 2009).

Monitoring through food attractions is considered important because it is directly related to the primary instinct of these insects, a hypothesis would be that females need protein compounds to reach their sexual maturity (Nakano et al., 1981). The most commonly used food attractions are sugarcane molasses, fruit juice, brown sugar or pheromones (Nascimento \& Carvalho, 1998).

In studies conducted by France et al. (2009), Neoleucinodes elegantalis (Lepidoptera: Crambidae) adults responded differently to the assessed attractions and parameters, with greater emphasis on sucrose and honey. Thus, it is expected that attractive baits with food stimulants make pest management by manipulating behavior an efficient tool and contribute to reduce the amount and increase the efficiency of pesticides applied in agricultural production systems. However, few studies on the use of attractive baits in the management of lepidoptera have been developed, and the greatest number of studies and information related to the use of baits in the management of diptera, such as fruit flies (Potts, 1999).

The baits may play a very important role in attracting insects, especially females responsible for reproduction. Research on the time to start and duration that adults spend to feed on these baits is fundamental, since it defines the amount of ingested product, potential for contamination and deleterious effect on the species (Potts, 1999; Borges et al., 2015).

Two main factors that should be considered in the choice of attractions for the formulation of toxic baits are: the attractive capacity, favoring the approach of the insect to the bait, and the phage stimulant effect or food response, increasing the consumption of bait that results in intoxication of individuals (Vargas et al., 2002; Pelz et al., 2005). The development of tactics for pest management based on manipulation of behavior has been very promising (Cook et al., 2007; Witzgall et al., 2008, 2010). Chemical substances involved in behavioral management, such as food stimulants and semi-chemicals, can become excellent auxiliary tools in pest control (Fosrter \& Harris, 1997).

The use of baits is an important tool in IPM, especially in decision making to control certain pests. However, with toxic baits a mortality factor is added, where a synthetic insecticide is usually used (Arruda-Gatti \& Ventura, 2003).

Papa et al. (2003) report the use of lures with insecticide cartape, associated with sugar, were effective in controlling adults of the pink cotton caterpillar, Pectinophora gossypiella (Lepidoptera: Gelechiidae). In relation to other lepidoptera, there is an indication for the control of moths in cotton and soybean with the application of a mixture of molasses, water and methomyl insecticide applied to the plants in bands with distances between them (Gallo et al., 2002).

In the management of $N$. elegantalis in tomato cultivation, toxic bait applications can contribute to the reduction of insecticide use and production costs, as well as benefit the environment and natural enemies, compared to conventional insecticide application systems (France et al., 2009). One of the advantages of toxic lures is the benefit to the environment, because they have less influence on natural enemies, because they are not applied in the total planting area, compared to the use of chemical insecticides (Gravena \& Benvenga, 2003; Galli et al., 2004).

\section{Conclusions}

The honeydew $(10 \%)$ and Noctovi ${ }^{\circledR} 43 \mathrm{sb}$ food attractions were more efficient in relation to feeding time for both females and all adults of $S$. frugiperda.

The highest number of landings was observed in the Noctovi ${ }^{\circledR} 43 \mathrm{sb}$ treatment in females and total adults.

The active ingredients methomyl, lambda-cyhalothrin and spinosad associated with food attractiveness are promising toxic baits for the management of fall armyworm.

\section{References}

Abbott, W. S. (1925). A method of computing the effectiveness of on insecticide. Journal Economic Entomology, 18(2), 265-267. https://doi.org/10.1093/jee/18.2.265a

Arruda-Gatti, I. C., \& Ventura, M. U. (2003). Iscas contendo cucurbitacinas para o manejo de Diabrotica spp. Semina: Ciência Agrária, 24(2), 331-336 
Benvenga, S. R., Fernandes, O. A., \& Gravena, S. (2007). Tomada de decisão de controle da traça-do-tomateiro através de armadilhas com feromônio sexual. Horticultura Brasileira, 25, 164-169. https://doi.org/10.1590/ S0102-05362007000200007

Borges, R., Machota, Jr. R.., Boff, M. I. C., \& Botton, M. (2015). Efeito de Iscas Tóxicas sobre Anastrepha fraterculus (Wiedemann) (Diptera: Tephritidae). BioAssay, 10(3), 1-8. https://doi.org/10.14295/BA.v10.134

Carvalho, R. A., Omoto, C., Field, L. M., Williamson, M. S., \& Bass, C. (2013). Investigating the molecular mechanisms of organophosphate and pyrethroid resistance in the fall armyworm Spodoptera frugperda. PLoS One, 8(4), e62268. https://doi.org/10.1371/journal.pone.0062268

Casmuz, A., Juárez, M. L., Socías, M. G., Murúa, M. G., Prieto S., Medina S., Gastaminz, A. G. (2010). Revisión de los hospederos del gusano cogollero del maíz, Spodoptera frugiperda (Lepidoptera: Noctuidae). Revista de la Sociedad Entomológica, 69, 209-231.

Cook, S. M., Khan, Z. R., \& Pickett, J. A. (2007). The use of push-pull strategies in integrated pest management. Annual Review of Entomology, 52, 375-400. https://doi.org/10.1146/annurev.ento.52.110405.091407

Cruz, I. (2008). Manejo de pragas da cultura do milho. In J. C. Cruz, D. K. M. A. R. Monteiro, \& P. C. Magalhães (Eds.), A cultura do milho (pp. 303-362). Sete Lagoas, Embrapa Milho e Sorgo.

Cruz, I., Figueiredo, M. L. C., \& Silva, R. B. (2010). Monitoramento de Adultos de Spodoptera frugiperda (J. E. Smith) (Lepidoptera: Noctuidae) e Diatraea saccharalis (Fabricius) (Lepidoptera: Pyralidae) em Algumas Regiões Produtoras de Milho no Brasil (Documentos/Embrapa Milho e Sorgo, 93, p. 42). Sete Lagoas: Embrapa Milho e Sorgo.

Cruz, I., Valicente, F. H., Viana, P. A., \& Mendes, S. M. (2013). Risco potencial das pragas de milho e de sorgo no Brasil (Boletim técnico, 150, p. 40). Sete Lagoas, EMBRAPA MILHO E SORGO.

Foster, S. P., \& Harris, M. O. (1997). Behavioral manipulation methods for insect pest-management. Annu. Rev. Entomol., 42, 123-146. https://doi.org/10.1146/annurev.ento.42.1.123

França, S. M., Oliveira, J. V., Picanço, M. C., Lôbo, A. P., Silva, E. M. S., \& Gontijo, P. C. (2009). Seleção de atrativos alimentares e toxicidade de inseticidas para o manejo da broca-pequena-do-tomateiro. Pesquisa Agropecuária Brasileira, 44(6), 561-568. https://doi.org/10.1590/S0100-204X2009000600003

Galli, J. C., Senô, K. C. A., \& Cividanes, F. J. (2004). Dinâmica populacional de crisopídeos (Neuroptera: Chrysopidae) associados a pomares de goiaba Psidium guajava L. com dois sistemas de pulverização de fenthion. Bol. San. Veg. Plagas, 30, 197-202.

Gallo, D., Nakano, O., Silveira Neto, S., Carvalho, R. P. L., Baptista, G. C., Berti Filho, E., ... Omoto, C. (2002). Entomologia Agrícola (p. 920). Piracicaba: FEALQ.

Gravena, S., \& Benvenga, S. R. (2003). Manual prático para manejo ecológico de pragas do tomate (p. 144). Jaboticabal: Gravena ManEcol.

Nakano, O., Silveira Neto, S., \& Zucchi, R. A. (1981). Entomologia econômica (p. 314). Piracicaba: Ceres.

Nascimento, A. S., \& Carvalho, R. S. (1998). Pragas da mangueira. In R. B. Sobrinho, J. E. Cardoso, \& F. C. O. Freire (Eds.), Pragas de fruteiras tropicais de importância agroindustrial (pp. 155-167). Brasília: EMBRAPA/SPI.

Nascimento, A. S., Carvalho, R. S., \& Malavasi, A. (2000). Monitoramento populacional. In A. Malavasi \& R. A. Zucchi (Eds.), Moscas-das-frutas de importância econômica no Brasil: Conhecimento básico e aplicado (pp. 109-112). Ribeirão Preto: Holos.

Nation, J. L. (2002). Nutrition. In J. L. Nation (Ed.), Insect physiology and biochemistry (pp. 65-87). Boca Raton: CRC Press.

Papa, G., Celoto, F. J., Takao, W., \& Prado, E. P. (2003). Efeito da isca cartap + açúcar sobre adultos lagarta rosada, Pectinophora gossypiella (Saunders, 1844) (Lepidoptera: Gelechiidae) (Embrapa Algodão. Documentos, 118, p. 4). Congresso Brasileiro de Algodão. Goiânia: Fundação GO.

Pelz, K. S., Isaacs, R., Wise, J. C., \& Gut, L. J. (2005). Protection of fruit against infestation by apple maggot and blueberry maggot (Diptera: Tephritidae) using compounds containing spinosad. J. Econ. Entomol., 98, 432-437. https://doi.org/10.1093/jee/98.2.432

Potts, L. (1999). Feeding stimulants and semiochemicals as pest management tools. Retrieved from http://www.colostate.edu/Depts/Entomology/courses/en507/papers_1999/potts.htm 
Silva, M. H., Camargos, G. S., Jardim, P. M. V., Andrade, R. S., Rezende, J. M., Albernaz-Godinho, K. C., \& Czepak, C. (2016). Ação do atrativo alimentar para mariposas Noctovi $^{\circledR}$ sobre o parasitismo de Trichogramma pretiosum Riley (Hymenoptera: Trichogrammatidae). Anais XXVI Congresso Brasileiro de Entomologia; IX Congresso Latino-americano de Entomologia (XXVI CBE/IX CLE). Maceió, Alagoas, Brasil.

Vargas, R. I., Miller, N. W., \& Prokopy, R. J. (2002). Attraction and feeding responses of mediterranean fruit fly and a natural enemy to protein baits laced with two novel toxins, phloxine B and spinosad. Entomol. Exp. Appl., 102, 273-282. https://doi.org/10.1046/j.1570-7458.2002.00948.x

Witzgall, P., Kirsch, P., \& Cork, A. (2010). Sex Pheromones and Their Impact on Pest Management. Journal of Chemical Ecology, 36, 80-100. https://doi.org/10.1007/s10886-009-9737-y

Witzgall, P., Stelinski, L., Gut, L., \& Thomson, D. (2008). Codling Moth Management and Chemical Ecology. Annu. Rev. Entomol., 53, 503-522. https://doi.org/10.1146/annurev.ento.53.103106.093323

Wyatt, T. D. (1997). Method in studying insect behaviour. In D. R. Dent, \& M. P. Walton (Eds.), Method in ecological and agricultural entomology (pp. 27-56). New York: CAB Internacional.

Zarbin, P. H. G., \& Rodrigues, M. A. C. M. (2009). Feromônios de insetos: tecnologia e desafios para uma agricultura competitiva no Brasil. Química Nova, 32(1), 722-731. https://doi.org/10.1590/S0100-40422009 000300016

Zenker, M. M., Specht, A., \& Corseuil, E. (2007). Estádios imaturos de Spodoptera cosmiodes (Walker) (Lepidoptera, Noctuidae). Revista Brasileira de Zoologia, 24(1), 99-107. https://doi.org/10.1590/S0101-817 52007000100013

\section{Copyrights}

Copyright for this article is retained by the author(s), with first publication rights granted to the journal.

This is an open-access article distributed under the terms and conditions of the Creative Commons Attribution license (http://creativecommons.org/licenses/by/4.0/). 